\title{
Free Movement of Workers and the Self-employment issue. The Case of The "Polish Plumber" in France
}

Free Movement of People offers new opportunities for European Union citizens migrating into the single market. This does however, bring controversy and public debates. This paper's focus is placed on the "Polish plumber" case - a symbol of social dumping. We study the European Union effects on the self-employment practices through the experience of Polish immigrants employed in low-skilled occupation in France. First, the reasons for setting up in France and the differences between labour conditions before and after the opening of the French labour market to Polish Immigrants are examined. The paper then discusses the self-employment career trajectories of Polish workers in France. Finally, we examine the impact that the Free Movement of Workers has on low-skilled occupations and the immigrants' adaptation and integration into host society.

Keywords: Free Movement of Workers, Immigrant worker, low-skilled occupations, self-employment, stereotypes

The European Union integration process has been driven by the implementation of measures that break down economic barriers in the Internal Market. Free Movement of people within the European Union territory is considered one of the founding principles of this process (Treaty establishing the European Economic Community: Article 45) and an attribute of European Union citizenship (EU Charter of Fundamental Rights: Article 45). Therefore the principle of Free Movement of Workers offers new opportunities to European Union citizens and

${ }^{1}$ Contact: Małgorzata Patok, malgorzata.patok@gmail.com 
their family members including the right to work and to reside in another Member State. Those workers should be treated on an equal footing with local citizens regarding recruitment, remuneration or training measures, as well as social and tax benefits (Directive 2004/38/EC).

As current debates around economic, migration and security crises show, the European Union integration process does not take place without controversy. Crisis reveals anxieties observable in all Member States where the question of immigrant workers is discussed widely. This is not a new phenomenon. The case of the "Polish plumber", which has evolved into a symbol of social dumping, is one such example. Coined in France in 2005 on the eve of the referendum on the Treaty establishing a Constitution for Europe, this stereotype referred to the immigrant workers seeking easily accessible jobs to improve their life situation (Patok 2015: 150). This high-tension debate was linked with the project of Bolkestein Directive on services and it has appeared due to reaction to fears about economic migrants flooding Europe's Old labour markets (Directive 2006/123/CE). In response, Poland launched a humorous campaign addressed to French citizens. This riposte has achieved success in French media and paradoxically the Polish immigrant worker became an advertisement (Patok 2017: 96-97).

The stereotype of the "Polish plumber" is my point of departure in the presented analysis of Polish workers in France. It is worth taking into consideration the deficiency of research regarding this group of Polish immigrants when compared to various scientific analyses of the previous waves of influx to France. Following Polish accession to the European Union in 2004, the Polish workers community in France differs from the three preceding migration waves: The Great Emigration after the November and January Uprising (Pezda 2001: 167-177), a large economic migration in the interwar period (Paczkowski 1979: 17) or political and economic migration from communist Poland after World War II (Śladkowski 2001: 15). Today France is not the main destination for Polish economic immigration. Also, the current community of Polish immigrants constitute rather a small fraction comparing to other groups of immigrants in France (Główny Urząd Statystyczny 2016). It is interesting to note that it is distinctively feminized when referring to the previous Polish waves of immigrations (Institut national de la statistique et des études économiques 2013).

In the context of economic and political challenges that Europe is facing nowadays, a question arises on the Free Movement of Workers effect on the integration process. This paper determines who exactly is the Polish immigrant worker and how she or he integrates and adapts her or himself to the French labour market. I looked into the Free Movement of Workers, following Poland's accession to the European Union in 2004, to understand the new situation of Polish workers in France. My main focus was examining the experience of low-skilled immigrants working under different arrangements including self-employment. Last but not 
least, the goal was to determine the impact that the Free Movement has not only on immigrants' career trajectories and entrepreneurships, but also on the integration process in the host country.

The first part of the paper is focused on the methodology and the study group. The next part deals with the results of the research. First, I will present Polish worker's motivations for migrating to France. The study will continue with the analysis of Polish immigrant's situation in the French labour market after 2004. Finally, I will examine the relation between self-employment and entrepreneurial activities of Polish workers in France. In conclusion, I will try to understand the impact the Internal Market Freedoms have on the immigrants' adaptation and integration into host society.

\section{Methodology and the study group}

This research was conducted in a Paris metropolitan area from 2011 to 2016, in a qualitative approach of Grounded Theory (Glaser \& Strauss 2006). The study is based on a corpus of 60 semi-structured interviews with Polish immigrant workers, through dozens of informal conversations, as well as via participant observation at their workplaces and homes. I also carried out observations in a public space frequented by the Polish community like Polish or French administration offices, Polish parishes and shops or public transport.

Non-probability sampling (Babbie 2007: 204-208) was used in order to choose the studied group within the population of low-skilled immigrants working in France (Table 1 and Table 2). By the category of low-skilled occupations I mean non-managerial positions that do not require higher education and are low placed on the social prestige scale. Polish immigrants are also employed in highly qualified managerial roles, though they involve a different analysis, and this is not the goal of this study. First, I have randomly selected groups of six unrelated low-skilled Polish workers. Then, each person indicated two or three other Polish immigrants from her or his labour environment who met the criteria of my target group. Selection of following respondents has been led according to the same rule. Finally, the study sample consists of 28 men and 32 women in working age.

The purpose was to create a various group of Polish immigrant workers who were diverse in their residence term and arrival moment, both before and after the Polish accession to the European Union structures in 2004. I also took into consideration the immigrant's age and the declared level of French proficiency. The diversity of these actors has allowed me to compare the experiences of Polish immigrant low-skilled workers in France. My main purpose was to understand the process of their adaptation and integration into the host state labour market, and not to represent the entire Polish immigrants in France or all low-skilled Polish 


\begin{tabular}{|c|c|c|c|c|c|c|c|c|c|c|c|c|c|c|c|c|c|c|}
\hline \multirow{7}{*}{ 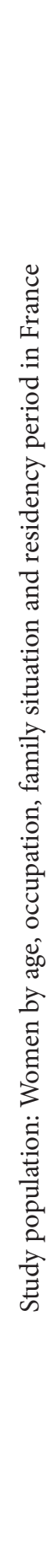 } & 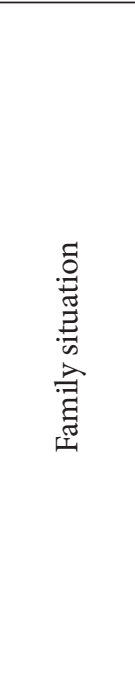 & 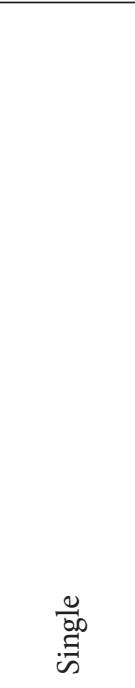 & $\begin{array}{l}\frac{0}{\infty 0} \\
\stackrel{\varpi}{\infty}\end{array}$ & 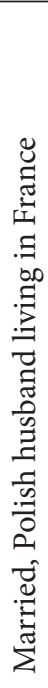 & $\begin{array}{l}\stackrel{0}{0} \\
\stackrel{0}{0}\end{array}$ & 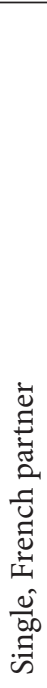 & 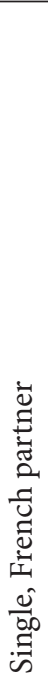 & 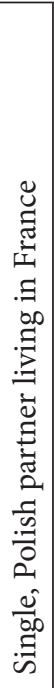 & 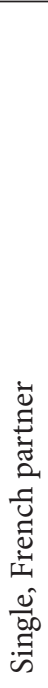 & 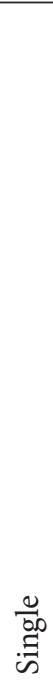 & 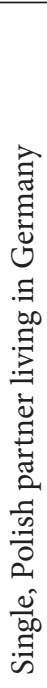 & \begin{tabular}{l}
$\stackrel{0}{\infty}$ \\
$\cong$ \\
\hdashline
\end{tabular} & 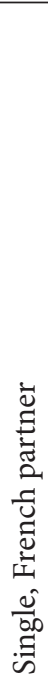 & 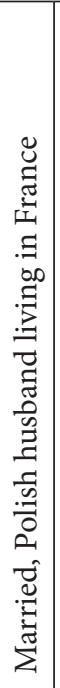 & 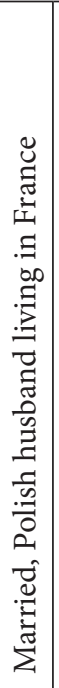 & 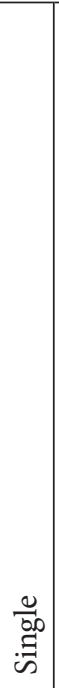 & 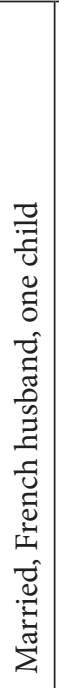 & 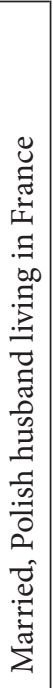 \\
\hline & 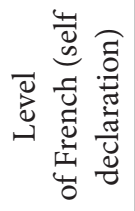 & 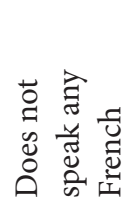 & 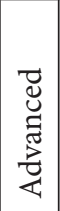 & 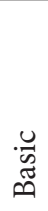 & 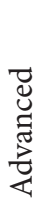 & 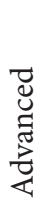 & 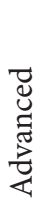 & 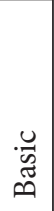 & 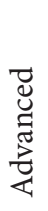 & 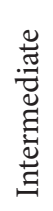 & 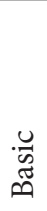 & . & 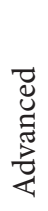 & 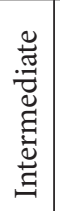 & 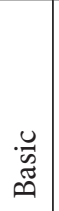 & 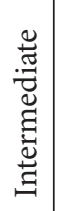 & 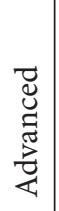 & 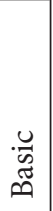 \\
\hline & 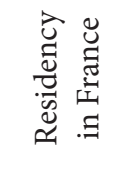 & $\begin{array}{l}\text { n } \\
\stackrel{ \pm}{ \pm} \\
0 \\
\vdots \\
0\end{array}$ & 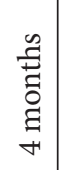 & 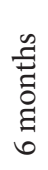 & 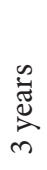 & 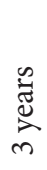 & 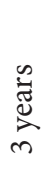 & 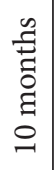 & 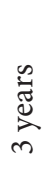 & \begin{tabular}{l}
0 \\
\multirow{0}{0}{} \\
0 \\
$\vdots$ \\
0 \\
0
\end{tabular} & 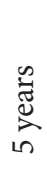 & $\underset{\beth}{\stackrel{\Xi}{\Xi}}$ & 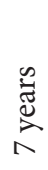 & 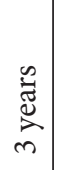 & 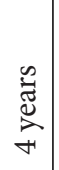 & 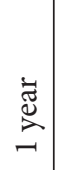 & 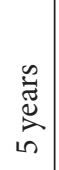 & 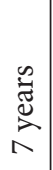 \\
\hline & 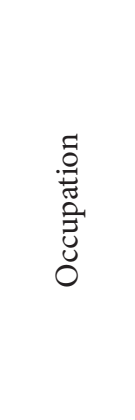 & 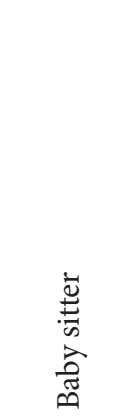 & 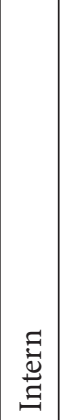 & 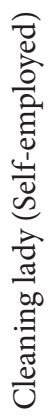 & 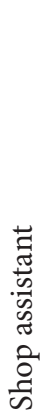 & 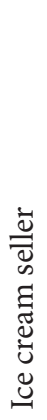 & 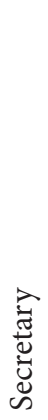 & 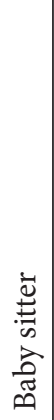 & 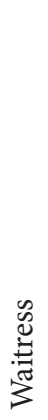 & 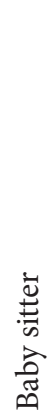 & 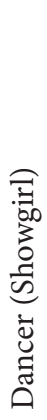 & 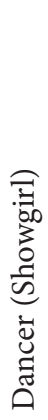 & 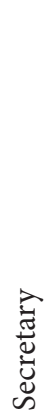 & 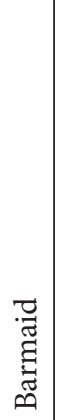 & 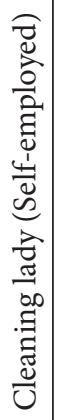 & 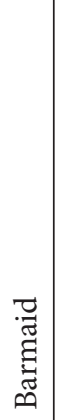 & 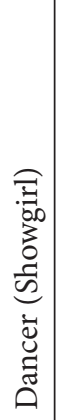 & 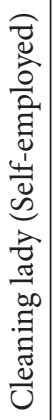 \\
\hline & $\stackrel{\infty}{4}$ & 고 & ○ & $\vec{\sim}$ & ป & $\ddot{\sim}$ & $\stackrel{\sim}{\sim}$ & $\stackrel{2}{\sim}$ & $\stackrel{20}{\sim}$ & $\stackrel{\sim}{\sim}$ & $\widehat{\curvearrowright}$ & $\widehat{\curvearrowright}$ & $\stackrel{\sim}{\sim}$ & $\stackrel{\infty}{\sim}$ & $\stackrel{\infty}{\sim}$ & શે & ి & ஜे \\
\hline & 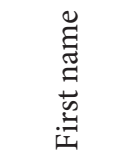 & 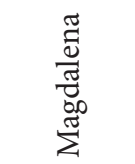 & 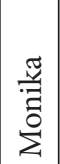 & 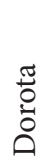 & $\begin{array}{l}\text { 䓂 } \\
\stackrel{0}{\Sigma}\end{array}$ & 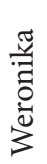 & $\begin{array}{l}\frac{\pi}{0} \\
\frac{\pi}{\pi} \\
\frac{0}{0} \\
\frac{0}{2}\end{array}$ & 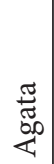 & 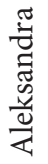 & 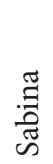 & م్య & 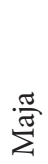 & 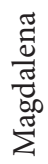 & 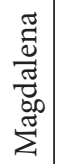 & 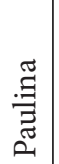 & 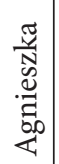 & 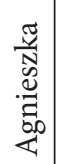 & 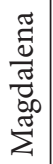 \\
\hline & $\stackrel{\circ}{Z}$ & $\neg$ & $N$ & $m$ & 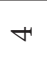 & in & 6 & $\wedge$ & $\infty$ & $a$ & 으 & $\exists$ & 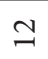 & $\stackrel{m}{=}$ & \pm & $\stackrel{20}{-1}$ & 0 & 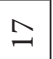 \\
\hline
\end{tabular}




\begin{tabular}{|c|c|c|c|c|c|c|c|c|c|c|c|c|c|c|}
\hline 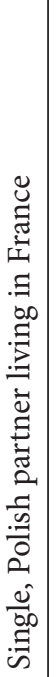 & 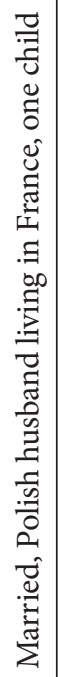 & 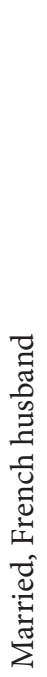 & 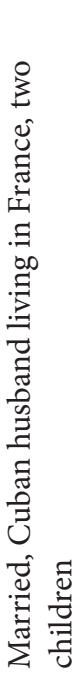 & 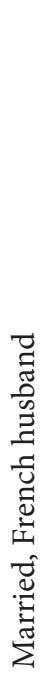 & 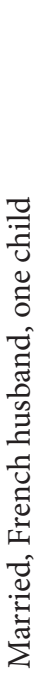 & 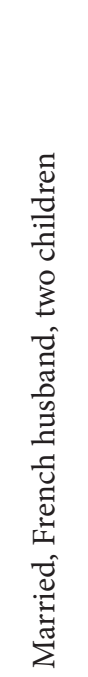 & 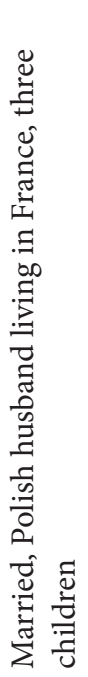 & 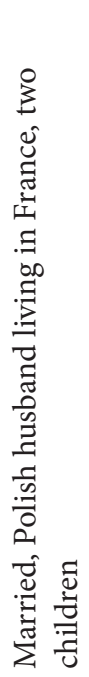 & 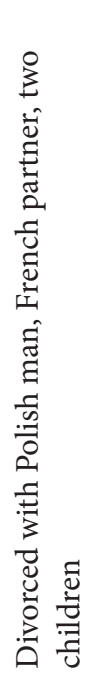 & 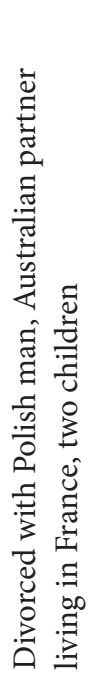 & 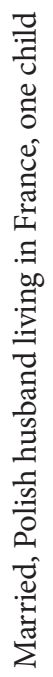 & 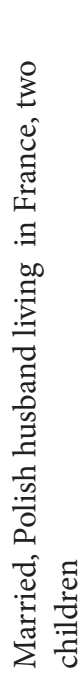 & 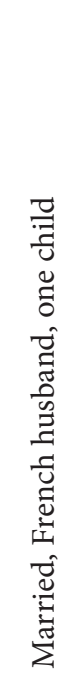 & 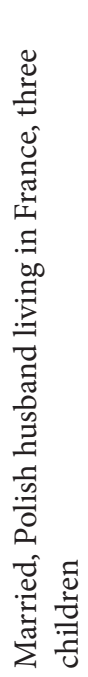 \\
\hline 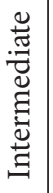 & 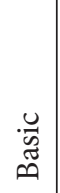 & 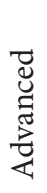 & 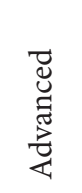 & 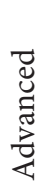 & 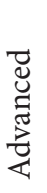 & 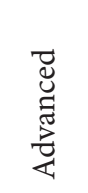 & 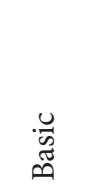 & 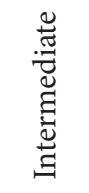 & 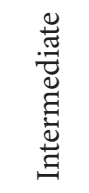 & 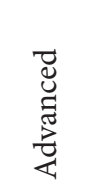 & 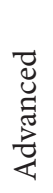 & 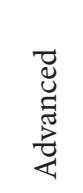 & 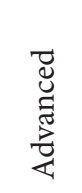 & 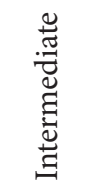 \\
\hline 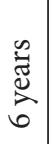 & 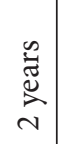 & 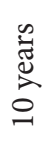 & $\underset{\text { in }}{\stackrel{\infty}{ٍ ँ ~}}$ & $\begin{array}{l}\stackrel{\infty}{\tilde{J}} \\
\stackrel{0}{0}\end{array}$ & 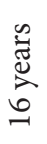 & 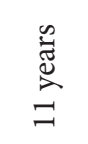 & 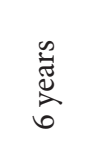 & 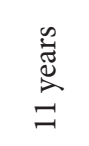 & 荧 & 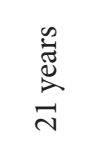 & $\begin{array}{l}\stackrel{\infty}{\Xi} \\
\stackrel{\Delta}{\Delta} \\
\vec{\sim}\end{array}$ & 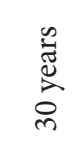 & 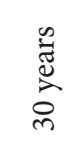 & 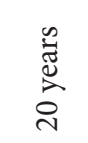 \\
\hline 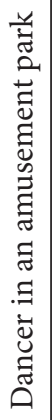 & 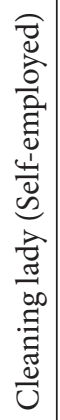 & 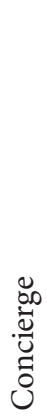 & 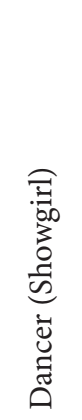 & 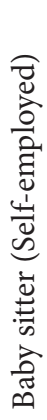 & 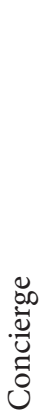 & 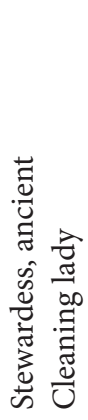 & 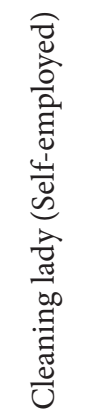 & 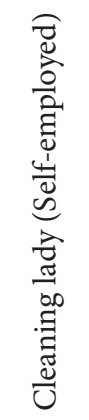 & 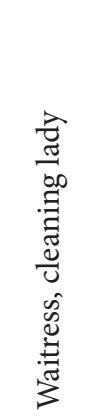 & 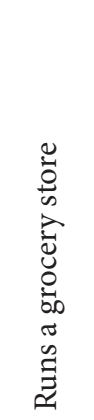 & 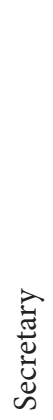 & 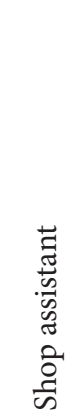 & 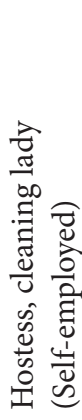 & 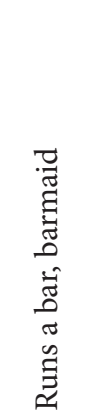 \\
\hline m & $\vec{m}$ & $m$ & $\ddot{n}$ & m & $\hat{n}$ & $\hat{n}$ & $\hat{n}$ & $\stackrel{\infty}{m}$ & $\stackrel{\infty}{n}$ & भे & $\bar{n}$ & L & ம & 이 \\
\hline 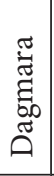 & 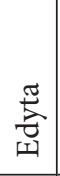 & 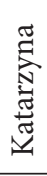 & $\begin{array}{l}\text { Iే } \\
\stackrel{\Xi}{\Xi} \\
\stackrel{0}{0}\end{array}$ & 营 & 芯 & 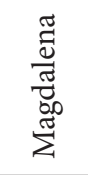 & 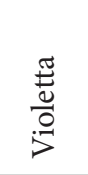 & 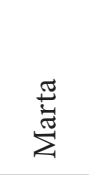 & 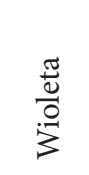 & 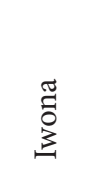 & 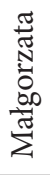 & 节 & 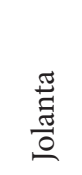 & 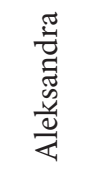 \\
\hline$\stackrel{\infty}{\sim}$ & $\curvearrowright$ & 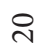 & $\vec{\sim}$ & సี & U & $\underset{\sim}{ }$ & $\stackrel{\mathscr{n}}{\sim}$ & $\stackrel{\sim}{\sim}$ & $\widehat{\Delta}$ & $\stackrel{\infty}{\sim}$ & ঐิ & లి & $\vec{m}$ & లె \\
\hline
\end{tabular}




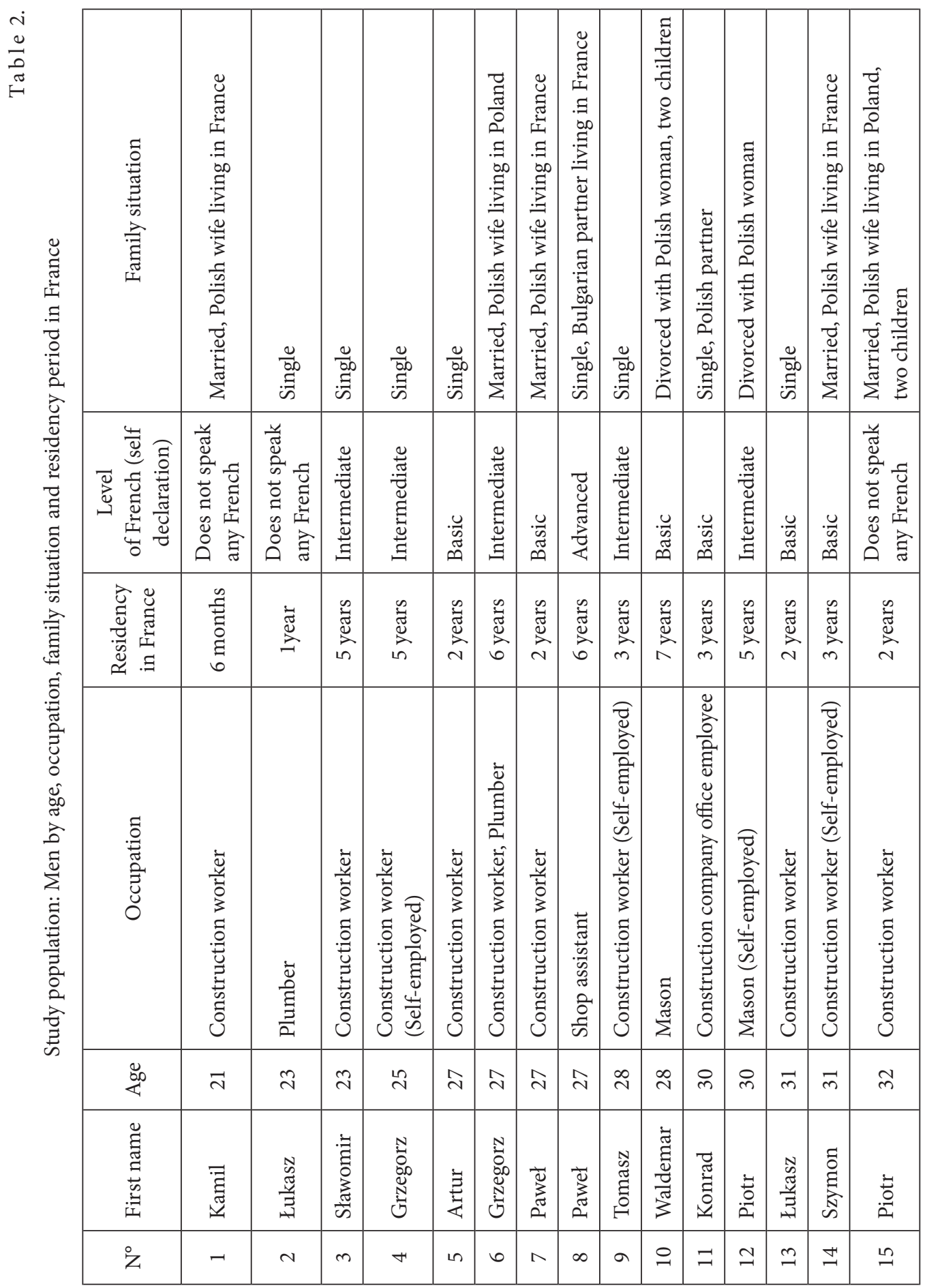




\begin{tabular}{|c|c|c|c|c|c|c|c|c|c|c|c|c|}
\hline 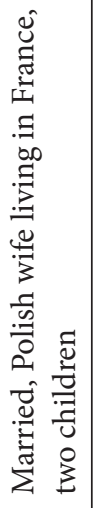 & 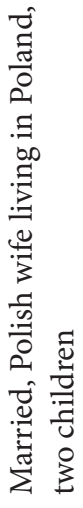 & 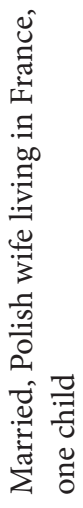 & 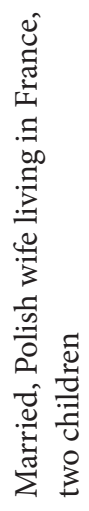 & 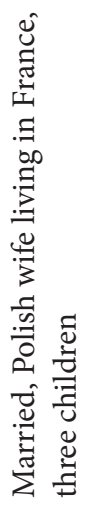 & 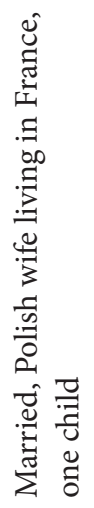 & 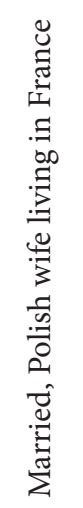 & 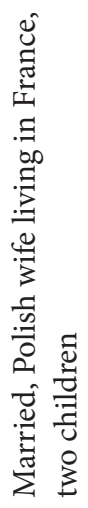 & 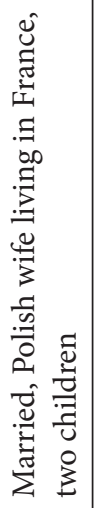 & 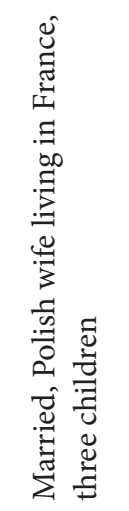 & 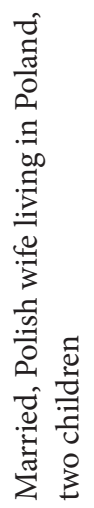 & 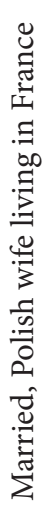 & 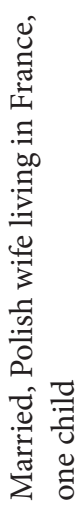 \\
\hline 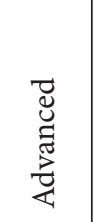 & 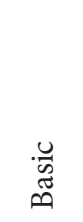 & 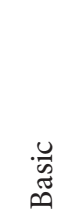 & 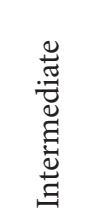 & 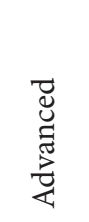 & 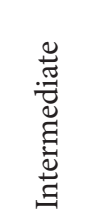 & 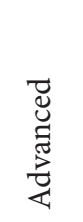 & 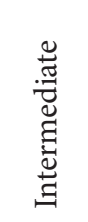 & 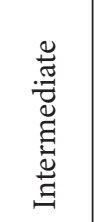 & 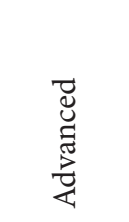 & 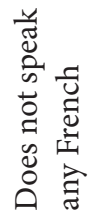 & 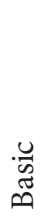 & 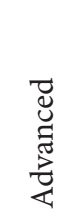 \\
\hline $\begin{array}{l}\stackrel{\infty}{\tilde{\Xi}} \\
\stackrel{\overbrace{}}{0}\end{array}$ & 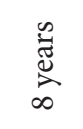 & $\begin{array}{l}\stackrel{\infty}{\tilde{J}} \\
\stackrel{2}{\Delta}\end{array}$ & 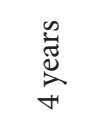 & 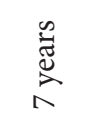 & 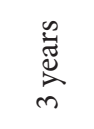 & 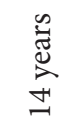 & 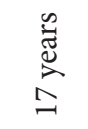 & 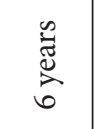 & 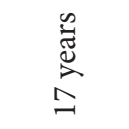 & 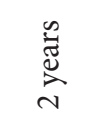 & 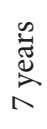 & 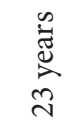 \\
\hline 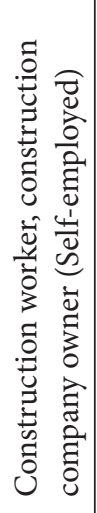 & 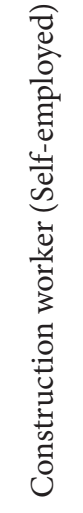 & 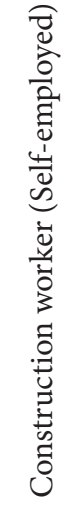 & 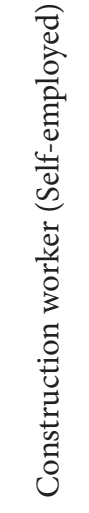 & 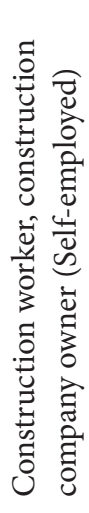 & 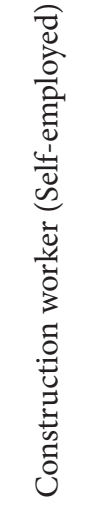 & 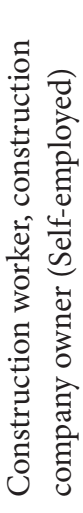 & 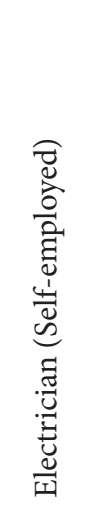 & 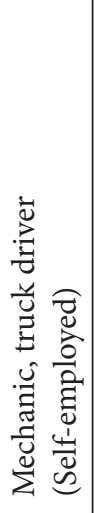 & 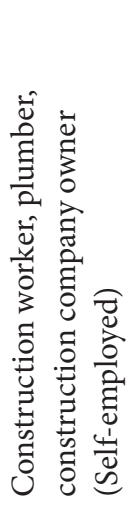 & 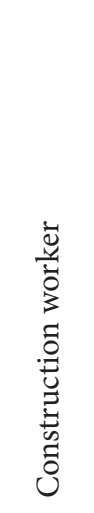 & 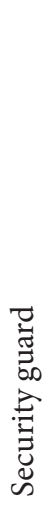 & 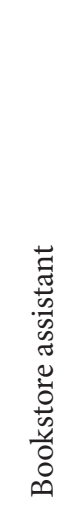 \\
\hline$m$ & $\stackrel{n}{n}$ & ల) & b & $\stackrel{\infty}{m}$ & $\stackrel{\infty}{m}$ & $\stackrel{\infty}{n}$ & $\stackrel{\infty}{m}$ & $F$ & $\stackrel{\infty}{+}$ & $\stackrel{\infty}{+}$ & in & 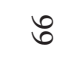 \\
\hline 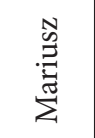 & $\stackrel{\overrightarrow{0}}{\circ}$ & 莒 & 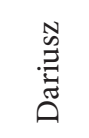 & 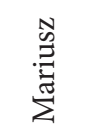 & 㫕 & $\begin{array}{l}\overrightarrow{\widetilde{\varpi}} \\
\text { こ्ञ }\end{array}$ & 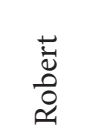 & 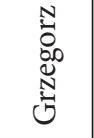 & 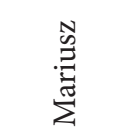 & 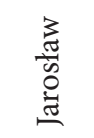 & 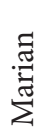 & $\bar{\Xi}$ \\
\hline$\stackrel{0}{\sim}$ & $\bumpeq$ & $\stackrel{\infty}{\sim}$ & $\curvearrowright$ & 구 & $\vec{\sim}$ & $\widetilde{\sim}$ & $\ddot{\sim}$ & $\stackrel{\sim}{\sim}$ & $\stackrel{\mathscr{n}}{\sim}$ & $\stackrel{\sim}{\sim}$ & $\sim$ & $\stackrel{\infty}{\sim}$ \\
\hline
\end{tabular}


workers in Paris. Finally, I would like to point out that as Polish economic immigrants in France had very active working days and limited free time, interweaving this group was a challenge.

In this research, to understand the integration process I traversed two theoretical frameworks. Firstly, I analysed both the relations between Polish immigrant workers and the French society e.g. colleagues, employers and customers. For this goal, I applied the concepts from symbolic interactionism (Becker 2009; Goffman 1996). Secondly, I referred to methodological individualism to examine choices made by immigrant workers in their professional life (Crozier \& Friedberg 1977; Boudon 1983).

\section{The results of the research}

\section{The origins and motivations for immigrating to France}

Following the political changes in Poland in the early 90s, the migration patterns for the greater number of Polish immigrants of the lower and middle classes are primarily economic ones. An economic migration is also a choice that in the current geopolitical circumstances does not exclude the option of a return to ones motherland (Bera 2011: 18). In terms of destinations for Polish immigrants, France ranks seventh, compared to other European Union countries such as Britain, Germany, Ireland, the Netherlands, Italy and Spain (Główny Urząd Statystyczny 2016). Therfore why do Polish workers choose to live in France today? Are they really ready to work for a lower wage as the stereotype of the "Polish plumber" initially insinuated? The interviews clearly show that the answer is based on three different types of motivations for arrival in France.

For the first type of immigrant worker the fact of living and working in France is not the result of a carefully thought-out life and career project. Their arrival is rather unplanned and the Polish worker had never thought of immigrating to France. The French language and especially the French labour market were totally unknown to her or him. While seeking a job in Poland, the immigrant finds the possibility to work in France and takes it. In the study group it was the case of unemployed family fathers or mothers, as well as singles trying to change their economic and professional situation. This type of immigrant worker arrives alone and has no family or friendship circles in France. Her or his motivation to leave Poland comes from the difficulties to find a job in their native country. In this case, the beginning of immigration is hard and a struggle. The immigrant worker is facing not only new living conditions alone, but also a day-to-day reality in her or his workplace. A strong economic motivation leads to accept a job bellow the immigrant's qualifications. The situation of Konrad, a 30 year-old construction 
worker living in France for 3 years, will help illustrate this scenario. This immigrant, graduated from the Polish University of Technology has agreed to work in a low-skilled occupation in France because of language barriers: "[...] working in construction means foremost working with people. You have to explain things or manage the workers. Really, it is a diplomatic work. If I do not speak the language I do not work at all. In the beginning, I was working as a "to do everything" man, so I've been doing the things that are not important at all. I didn't develop myself. I accepted this situation, but I did not feel well [...] ".

The second case of immigrant worker joins her or his family or friends already working in France, thus a network of contacts is rather established. This immigrant arrives because the work and accommodation are guaranteed. She or he is attracted by a better quality of life and higher wage opportunity. In the studied group, men employed in building construction and renovation exemplified this type of motivation. These workers came from little towns or villages like Waldemar, a 28-year-old mason, in France for 7 years: "[...] First, my mother came here [to Paris], then, my big sister came to work and my little sister was at school here. [...] I finished school and I've seen any job prospects, especially for a good salary, so I thought it would be better to leave, to learn a foreign language and to start working [...]". This case of immigrant also immigrates with their spouse or joins their partner in France. Magda, a 30-year-old married cleaning lady gives an illustration: "I came just for him, I didn't want to come here [...]. I do not want to live in France; I always knew that I wouldn't feel good in a foreign country. I've sacrificed myself to be and to live together. That's why I'm here. [...]". For this type of immigrant worker the decision to leave results from reflexion, especially in the case of persons already employed in Poland. However, immigration may be also a spontaneous choice for unemployed individuals looking for a job. The study clearly shows that the experience abroad is often different from expected immigration reality. In spite of the difficulties in France, the immigrant usually doesn't return to Poland. Rafał, a 38-year-old construction worker, in France for 14 years explains: "[...] at the very beginning, I wanted to return back to Poland because I had never worked in construction before. I was supposed to be a courier or deliveryman [...] but this work wasn't available. At this time the situation in Poland was difficult. I have borrowed some money from my mother and I have come here. I said to myself that if I am here, I had to work to give back that money. It was hard, but I started to work in construction".

The interviews clearly show that young female already having contact with the French language represented the third and the last type of migration motivations. It was a case of students usually working as baby-sitters and cultivating the myth of France renowned for its artistic heritage. In the study group these immigrants' aspiration was to integrate into French culture and to fulfil a profession in France. Immigration is part of a self-realization plan based on the desire to get closer to 
French culture. The departure of Poland is prepared well in advance including a job, such as waitress or baby-sitter, allowing contacts with French society and language. Sabina, a 26-year-old au pair caregiver, gives such an example: "[...] I've fallen in love with the French language and to leave, it was my dream. I have realized that one person from my family was in France so one day I called her to ask if she could help me. She had been looking for my job for six months and finally she called me. I've arrived on November 9th. I remember that date very well'. In the study group, this type of immigrant worker desires a new life, to change environment or to move to a bigger city. In consequence, the Polish worker takes a risk as she or he seeks the possibility of changing jobs.

In the study group, the migration patterns of low-skilled Polish workers in France are mainly economic. Nevertheless, the departure of the immigrant may not be the result of a carefully planned project. When seeking a job in Poland, the individual can find her or his employment opportunity in France. The immigrant network of weak ties (Granovetter 1973), such as people met by chance out of the immigrant's family and close friendship circles, plays an important role in this process (Patok 2013: 259). In all studied cases the immigrant worker stays despite the difficulties because a return could be considered as migratory failure in front of relatives in Poland.

\section{Polish worker's situation in the French labour market after 2004}

Since the opening of the French labour market in 2008, four years after Poland's accession to the European Union, the Polish worker is subject to similar administrative procedures as French citizens. The situation for both, the Polish immigrant and for her or his French employer has evolved. This part of the paper analyses the experience of immigrants from the study group in order to understand their current professional situation, not only in the field of law, but also from a social and economic point of view. Following the interviews, I can distinguish four major differences regarding Polish worker's situations in the French labour market before and after 2004.

First, illegal forms of employment have become less interesting. Legal employment guarantees social security and the recognition as an equal employee. However, the usual wage for a construction worker has fallen due to social charges. This is illustrated by Waldemar, a 28-year-old mason: "At the beginning I had been doing undeclared work, like most Polish workers have. [...] Before [2008] it wasn't so easy [...]. You weren't allowed to work if the boss couldn't prove that you were a wanted employee and that there wasn't a French unemployed worker for that position. Now, we have the opportunity to sign a contract and to start working the same day".

Second, the labour market opening ensures the Polish worker's legal rights in France. In the study group it is significant, especially for cleaning ladies and 
construction workers looking for a declared employment. Having a professional occupation is no more a necessity for a Polish economic immigrant planning to stay abroad, as it was before 2004 when a residence permit was an obligation. The experience of Polish workers from the study group also illustrates that now the law limits the employer's control and power. Grzegorz, a 41-year-old mechanic and lorry driver explains this situation: "People have no idea what the European Union means. [...] Before it was the employer who determined his own law and he could throw you out if you disobeyed his illegal rules. He could blackmail you. For us, this open border is something really beautiful. [...] We can change jobs without stress".

Third, according to opinions of workers from the study group, the attitudes of their Polish colleagues arriving after the French labour market opening - have changed. The interviews show that previously, the Polish immigrant wave was more motivated to work. A stay of Polish employees in France was limited before 2004, which had an impact on the motivation to work. The economic immigrant objective was to make and to save money or reside abroad for as long as possible. In the study group the Polish workers migrating before 2004 perceive the "new arriving" as more demanding or an even pretentious employee. An opinion of Rafał, a 38-year-old construction worker living in France for 14 years gives an illustration: "[...] I remember when I've arrived [to France]. At this time only a three-month stay was possible. I remember how much effort we'd been making. [...] I think it's about how important something is to you. [...] So it's a matter of motivation".

Finally, after 2004 the number of Polish immigrants in France has increased. In consequence, they feel more anonymous and their values are different from previous Polish immigration waves. Iwona, a 49-year-old vendor from a Polish store living in France for 21 years, explains her every day observation: "Before Polish immigrants were more honest. We have never verified the checks coming from Polish customers. [...] Since the borders are open, we have begun to have problems [...] we can no longer accept payment with a check. And this is only the fault of the new customers. The older clients, I mean this generation, who arrived previously was so honest. Those people were different". However Iwona also perceives the positive aspects of the Free Movement of Workers. The current situation of people arriving in France is more convenient. Consequently, their adaptation and integration process into the host society goes smoothly. Indeed, these newly arriving immigrants are considered as courageous in their professional choices, such as decisions regarding changing job. Iwona explains this situation: "New immigrants are more open-minded, more free. It seems, in my opinion, that they have more enterprising skills because they didn't have so many constraints in the beginning [of the migratory trajectory]. They assimilate quicker. It's easier to get documents, to get and to keep a job. This generation is more stress-free".

The research shows that Poland's accession into the European Union structures, and consequently the opening of the French labour market to the Polish workers 
had a significant impact on these immigrants' everyday life in France. The Polish worker has become equal as a subject of law comparing to her or his French colleague. Not only are the administrative procedures less complex for both the immigrant worker and her or his French employer, but also the Polish worker is more aware of her or his rights.

\section{Self-employment and the Polish immigrant's entrepreneurship in France}

Lauto-entreprenariat or le statut d'auto-entrepreneur is a self-employment system created in 2008 by François Fillon's government, under the presidency of Nicolas Sarkozy in order to stimulate French economy (Auto-entrepreneur.fr 2017). For individual entrepreneurs, a single fee proportional to their income has replaced all social contributions, as well as all taxes to simplify administration procedures. Consequently, if the entrepreneur has no revenues, she or he doesn't pay contributions (Droitfinaces.net 2017). Individual businesses have also become easy to initiate, to interrupt or to end via online registration (Planète Auto-entrepreneur 2017). Moreover, even if an individual is already employed, this system provides an opportunity to create a single company and to carry on an independent business. A part time or full time occupation can be combined with self-employment status. Job seekers, students or pensioners can also benefit from this system (Service Public 2017). Self-employment includes most activities, however some of them; such as agriculture, public administration and scientific or artistic literary production are excluded due to affiliation to a different tax system (Auto-entrepreneurs info 2017). It is also important to mention some critical aspects of self-employment in France. This system provokes layoffs during economic crises. Companies, rather than hiring, may illegally ask their employees to change their status to self-employed in order to reduce taxes. This leads to insecurity, which affects the employees' lives career negatively (Bonnard 2013).

The interviews from presented fieldwork clearly show that self-employment is an important form of legal activity of low-skilled Polish immigrants in France. In the research sample, 8 women and 14 men represent this form of professional activity. All self-employment cases were registered in France, so are subjects to French law. In the study group I can distinguish two types of self-employment activities of Polish workers, such as the cleaning ladies and baby-sitters, as well as the construction workers.

This paper's focus is on different stages of the professional career of the Polish self-employed immigrants. The career concept is understood here as the succession of occupations held by the individual (Hughes 1937: 408). A professional career consists, objectively of a series of status passages and clearly defined offices, and subjectively of the individual perception of her or his reality as a worker (Idem). The professional career also can be studied as a variation of organisations, institutions and formal or informal networks (Hall 1948: 327). In the study of career trajectories 
as a process, I refer to synchronous and sequential models analysis (Becker 2009: 45). It stresses the importance of changes regarding the transformations of the Polish immigrants' motivation and desire over time. The interviews show that the Polish immigrant working in France as self-employed goes through several phases of professional development. Consequently, I distinguish three models for a career: Stagnated Model, The "Jack of all trades" Model and Developing Career Model.

First, I focus on self-employed cleaning ladies and baby-sitters. In the study group only woman represented them. In this case self-employment provides an opportunity to legally perform professional activity in the French labour market. Before the opening of French labour market for the Polish workers, a job position in this domain was undeclared. Today, the procedure takes place at the request of the employer interested in maid tax write-off. As a result an immigrant woman can easily access healthcare and social welfare in France, which is attractive because it affects her sense of security and stability in the host country. It is also possible to work for individual families and not only for large companies, which gives autonomy in setting up working hours.

In this case the Polish worker's career stages represent a Stagnated Model. The immigrant has the same professional occupation since her arrival, so the skills and the profession do not change, in contrast to contact networks. An extensive number of customers' demands let a self-employed immigrant woman choose the most conforming or lucrative offers. Marta, a 38-year-old cleaning lady working in France for 11 years, explains that today she works mostly for families with whom she has good relationships. She stresses the importance of her occupation's flexibility allowing her to take care of her two boys: "Now I work for people I like. I like their homes. First we drink coffee and then they go to work and I do my job. I'm not considered a housekeeper, but rather someone who helps. Now, I work solely for people who accept that I'm not available during school holidays [...]. Since I have children, I work for people who don't mind that I'm not working during holidays. It's not easy to find such people. Now I'm in a comfortable position where I get to choose who I work for (...)."

The construction workers represent the second type of self-employment activities of Polish immigrants in France in the study group. In this case, the immigrant is a man; he already knows the French labour market and his network of clients is developed. Consequently, self-employment results from a need of professional autonomy and a more lucrative income. As for non-French speaking self-employed immigrants, it's usually their neighbours or family members who manage the administrative procedures. It is worth noting that in the opinion of Polish workers, the professional activity as a subject of French law is more credible for French customers. The interviews let me distinguish two models of the career stages in the case of a self-employed construction worker.

Developing Career Model is my first example. The research shows that in this case professional trajectory progresses from young assistant to owner of a renovation 
company. Once employed, the worker goes through several stages. Beginning as an assistant, he gradually learns other skills, such as brickwork, electrics and plumbing to finally open his own business. It is worth mentioning that the Developing Career Model also exists in the case of women from the study group. It starts from baby-sitter or waitress then, after studies or professional courses, it evolves to secretary or stewardess. However, this case doesn't apply to self-employment's category. Artur, a 27-year-old construction worker living in France for 2 years explains the process of acquiring new skills in the construction domain: "[...] When I came to the firm, the other guys showed me how to work here [in France], how to do the job, which was different with [work in] Poland. In Poland I learned to run metal buildings construction, and there [in France], you have to learn everything: electrics, painting, etc. At the beginning I was only helping and then every year I learned something new. For example, I was asked to manage the construction of a wall and after that it was verified. If the result wasn't good, they showed me how to do it correctly. A half-year later, I proved that I know how to work and I started running my own business, which I was responsible for [...]".

Thus, the study demonstrates that when the immigrant worker manages different skills, he feels more independent and consequently he thinks about his own company. However, the self-employment decision results from various factors. The interviews show that several years of experience on the French market brings a requirement for new services. In this case, especially the Polish workers with financial family-related responsibilities decide to start their own business. Yet some immigrants arriving in France are already planning to work for their own account in the future. The interviews indicate that those men, usually young, improve their French prior, get professional experience and build a contact network. The experience of a 38 year-old self-employed construction worker, Rafał will help illustrate this scenario: "When there was an opportunity for working on my own, I decided to start my own business quickly. If I work for a French company, I have SMIC [The guaranteed minimum growth wage]. [...] and when you're working for someone [as employee] you are ordered to leave or not. You have to explain everything to everyone. Now it would be difficult for me to follow orders. When I met a few people I started doing some undeclared jobs; but you know, you need to show the accounts, so I've founded a Polish company. I've been running it for three years. Then, I decided to start a French business. Now I am working under self-employment and this is less complicated, mainly accountancy".

A "Jack of all trades" Model illustrates the second type of Polish self-employed construction worker in France. This case represents an immigrant having different occupations for many years, for example a mechanic and a lorry driver at the same time. The immigrant workers have several jobs in various sectors in order to carry out future personal projects, such as education, retirement or family house construction. The professional background of the immigrant worker is varied, but 
not continuous. The individual doesn't develop skills within a specific field, but he changes trades and sectors several times. This case of the self-employed worker is focused on financial benefits, new experiences and professional contacts with different people. The "Jack of all trades" Model is also a way to benefit efficiently from the stay on immigration. Moreover, it can be a transition between a current job and the main future professional occupation. In the study group the work requiring huge physical strength and dexterity was considered as short-term. Grzegorz, a 41-year-old circus artist living in Paris for 6 years, will illustrate this process: "It's clear that the first thing is to make money. We can always organise our time well. The work must be a pleasure, but it's also the opportunity to make money. Unfortunately, years pass and I need to have some financial security. When I was a 15 or 18 -year-old boy the circus was my passion. Well, right now... when I arrived [in France], I hadn't an opportunity for extra income. I was focused on artistic work. That was my goal. With time, I've been making friends and creating new contacts in many different places, so I could do more things [out of work in the circus]."

The research shows that the career trajectory of self-employed baby-sitters and cleaning ladies represents a Stagnation Model. These immigrants don't have a professional promotion, but they develop large networks within their domain. In this case self-employed worker feels free to choose among customers and job offers. In the study group the self-employed construction workers represent rather a Developing Career Model. The immigrant workers start as assistants and with time they open their own businesses. Finally, the self-employed Polish immigrant from the construction sector can also carry out several activities from different sectors. This is a case of the "Jack of all trades" Model. The self-employed immigrant representing this career model adapts and integrates into the French labour market in many segments if comparing to the two previous career models. Consequently, a "Jack of all trades" career trajectory allows an immigrant worker to compare various trades in the French labour market before he decides about their final occupation.

\section{Conclusion: Free Movement of Workers and Immigrant's Integration into the Host Society Labour Market}

Self-employment is an example of adaptation and integration of Polish lowskilled workers in the French labour market. On one hand, French customers confront Polish immigrants with different requirements. On the other hand, their versatility and determination allow them to quickly adapt to new demands from the French labour market. Certainly, the situation of the Polish immigrant regarding relations with French employer has improved due the Free Movement of Workers principle. The French client hiring a Polish worker has no longer to go trough the additional procedures. Moreover, the Polish worker is free to choose her or his 
customer, company and duration of stay abroad. It is important to stress that this consciousness of freedom is a key consequence of the Free Movement of Workers on the immigrant worker.

The French labour market opening has a strong impact on Polish immigrants' adaptation and integration into the host society. The studied trajectories of professional careers: Stagnated Model, Developing Career Model and the 'Jack of all trades" Model, clearly indicate that both legal recognition and equal rights to French citizens, encourage low-skilled Polish immigrants to perform and to register their professional activities as self-employed workers in France. In consequence, those immigrants not only assimilate to the French legal or administrative requirements regarding their professional segment, but also realize and integrate informal rules of the French labour market. Better is the Polish workers' understanding of their host society, where their professional decisions are more courageous. The immigrant workers, aware of their employee's rights and duties articulate their professional needs more openly to French employers and customers in all three studied models of career trajectories. Polish workers' sense of autonomy is more developed then before the French labour market opening.

Following the analysis of three professional career models of studied immigrants, I noticed that professional network is a significant aspect of Polish workers' adaptation and integration process in France. To have lucrative self-employment requires large contact networks, consequently an immigrant worker needs to establish relationships with the host society. The cleaning ladies and baby-sitters representing a Stagnated Career Model are focused on one segment of the labour market, where they develop a big number of customers usually families. In this case, culture of the host society is discovered through those families' domestic lifestyle. The construction workers looking for autonomy and lucrative income illustrate a Developing Career Model. They go through several stages of professionalization, adapting their services to the French market's demands and becoming more credible for French customers and employers. The "Jack of all trades" Career Model is a trajectory of different occupations, thus the immigrant worker adapts and integrates in many segments. A varied professional network allows comprehending many different aspects of the labour market. However, in the three studied models of career development, the most important factor of the adaptation and integration process is the selfemployed immigrants' plan for their future stay. If the Polish worker visualises his or her family life in France, he or she willingly adapts and integrates into the host society. In this case the immigrant is motivated to learn French, develop their professional career or contact network, as well as evolve into the French environment. At the same time, his or her relations with compatriots in France decrease to family or friendship circles for contacts with the host society (Patok 2016).

It is important to stress that self-employment is also a symbol of occupational prestige for studied workers. As economic immigrants employed in low-skilled jobs, 
the Polish workers have a strong need for recognition. They feel inferior in the eyes of their French employers or clients. At the same time, they notice that they can be recognized as good workers and entrepreneurs. This is a very important aspect of their identity as immigrants and it includes a sense of respect and dignity, which are key elements of the integration process. The economic activity, as well as a sense of entrepreneurship defines the migratory success. However, seeking the French society's recognition in labour activities confirms the stereotype of a ready to work immigrant.

The results of this research show that the European Union integration process is based on Internal Market Freedoms. The economic activity is changing the mentality of Polish immigrants towards the consciousness of equality and liberty. However, this consciousness of rights as European citizens is not established yet on the political level.

A question rises whether or not the adaptation and integration processes in the case of overqualified Polish employees with a principal status are the same as in the law-skilled workers case? Finally, how to reach the next level in the European integration process (beyond the economic level) and develop the awareness of the necessity of integration at the political level amongst the European Union citizens? The challenge is to rethink the European Union integration policies from the perspective of its main actors: the European Union's citizens.

\section{References}

Auto-entrepreneur.fr, http://www.auto-entrepreneur.fr/ [Accessed: 27.09.2017].

Auto-entrepreneurs info, Les professions et professionnels exlus du regime d'auto-entrepreneur, https://autoentrepreneurinfo.com/les-professions-et-professionnels-exclus-du-regime-dauto-entrepreneur [Accessed: 27.09.2017]

Babbie E. (2007), Badania społeczne w praktyce, translation A. Kłoskowska-Dudzińska, Warsaw: Wydawnictwo Naukowe PWN.

Becker H.S. (2009), Outsiders, Leétude de sociologie de la déviance, translation J.P. Briand and J.M. Chapoulie, Paris: Métailié.

Bera R. (2011), Aksjologiczny sens pracy a poczucie jakości życia młodych emigrantów polskich, Lublin: Wydawnictwo Uniwersytetu Marii Curie-Skłodowskiej.

Bonnard J. (2013), Auto-entrepreneurs: un bilan mitigé, une réforme limitée, Le Monde, http:// www.lemonde.fr/economie/article/2013/05/29/auto-entreprise-un-bilan-mitige-pour-unstatut-conteste_3419257_3234.html [Accessed: 27.09.2017].

Boudon R. (1983), La logique du social, Paris: Hachette.

Crozier M., Friedberg E. (1977), Lacteur et le système, Paris: Seuil.

Directive 2004/38/EC of the European Parliament and of the Council of 29 April 2004 on the right of citizens of the Union and their family members to move and reside freely within the territory of the Member States amending Regulation (EEC) No 1612/68 and repealing Directives 64/221/EEC, 68/360/EEC, 72/194/EEC, 73/148/EEC, 75/34/EEC, 75/35/EEC, 90/364/EEC, 90/365/EEC and 93/96/EEC.

Directive 2006/123/EC of the European Parliament and of the Council of 12 December 2006 on services in the internal market. 
Droit-finaces.net, Auto-entrepreneur : définition, statut et avantages http://droit-finances.commentcamarche.net/contents/622-auto-entrepreneur-definition-statut-et-avantages [Accessed: 27.09.2017].

Espace Auto-entrepreneur, Focus sur le statut d'auto entrepreneur 2017, [Accessed 27.09.2017] EU Charter of Fundamental Rights, Article 45.

Glaser B., Strauss A. (2006), Discovery of Grounded Theory, Strategies for Qualitative Research, Mill Valley: Sociology Press.

Główny Urząd Statystyczny (2016), Informacja o rozmiarach i kierunkach emigracji z Polski w latach 2004-2015, http://stat.gov.pl/obszarytematyczne/ludnosc/migracje-zagraniczne-ludnosci/informacja-o-rozmiarach-i-kierunkach-emigracji-z-polski-w-latach-20042015,2,9. html [Accessed: 27.09.2017]

Goffman E. (1996), Stigmate, Les usages sociaux des handicaps, translation A. Kihm, Paris: Les éditions de minuit.

Granovetter M.S. (1973), The Strength of Weak Ties, „American Journal of Sociology”, vol. 78, Issue 6, p. 1360-1380.

Hall O. (1948), A Stages of a Medical Career, „American Journal of Sociology”, vol. 53, Issue 5, p. 327-336.

Honneth A. (2000), La lutte pour la reconnaissance, translation P. Rusch, Paris: Gallimard, Folio essais.

Hughes E.C. (1937), Institutional Office and the Person, „American Journal of Sociology”, Vol. 43, Issue 3, p. 404-413.

Institut national de la statistique et des études économiques (2013), Pays de naissance des immigrés entrés en France en 2012 et part des femmes, https://www.insee.fr/fr/ statistiques/1281393\#titre-bloc-4 [Accessed: 27.09.2017]

Paczkowski A. (1979), Prasa i społeczność polska we Francji 1920-1940, Cracow: Ossolineum.

Patok M. (2013), Wzajemne relacje w środowisku polskich pracowników we Francji po przystąpieniu Polski do Unii Europejskiej, in: Lesińskia M., Okólski M. (eds.), Współczesne polskie migracje: strategie i skutki społeczne - reakcja państwa, Warsaw: Wydawnictwo Uniwersytetu Warszawskiego, p. 241-261.

Patok M. (2015), Stereotypy narodowe a normy i kontrola społeczna w środowisku polskich pracowników we Francji po 2004 roku, in: Zamecka J. (eds.), Normy, dewiacje i kontrola społeczna, vol. 16, Warsaw: IPSIR, p. 148-163.

Patok M. (2016), Economic Migration in the Context of the Internal Market of the EU: the Processes of Adaptation and Integration of Polish Workers in France », in: Martin C. et Marasova J. (eds.), Pour une Europe forte, rénover les structures économiques, entrepreneuriales, politiques, territoriales et éducatives, Bratislava: Wolters Kluwer, p. 239-250.

Patok M. (2017), Le stéréotype national: l'exemple de la peur du "plombier polonaise", in: Rouet G., Stoicheva M. (dir.), Identités, démocratie, frontières, Paris: L'Harmattan, p. 93-104.

Pezda J. (2001), Polska diaspora we Francji do 1870 roku, in: Walaszek A. (eds.), Polska diaspora, Cracow: Wydawnictwo Literackie, p. 167-177.

Planète Auto-entrepreneur, Comment déclarer votre activité d’auto-entrepreneur? http://www. planete-auto-entrepreneur.com/autoentrepreneur-1/comment-declarer-activite-1.html [Accessed 27.09.2017]

Service Public, Devenir auto-entrepreneur, https://www.service-public.fr/professionnels-entreprises/vosdroits/F23961[Accessed: 27.09.2017]

Śladkowski W. (1980), Emigracja polska we Francji 1871-1918, Lublin: Wydawnictwo Lubelskie. Treaty establishing the European Economic Community, Article 45. 